\title{
Lung scintigraphy for pulmonary embolism diagnosis in COVID-19 patients: a multicenter study.
}

Pierre-Yves Le Roux ${ }^{1}$, Pierre-Benoit Bonnefoy ${ }^{2}$, Achraf Bahloul ${ }^{3}$, Benoit Denizot ${ }^{4}$, Bertrand Barres ${ }^{5}$, Caroline Moreau-Triby ${ }^{6}$, Astrid Girma ${ }^{7}$, Amandine Pallardy ${ }^{8}$, Quentin Ceyrat ${ }^{9}$, Laure Sarda-Mantel ${ }^{10}$, Micheline Razzouk-Cadet ${ }^{11}$, Reka Zsigmond ${ }^{12}$, Cachin Florent $^{5}$, Gilles Karcher ${ }^{3}$, Pierre-Yves Salaun ${ }^{1}$

Affiliation for authors:

${ }^{1}$ Université Européenne de Bretagne, Université de Brest, EA3878 (GETBO) IFR 148, CHRU de Brest, Service de médecine nucléaire, Brest, France.

${ }^{2} \mathrm{CHU}$ de Saint-Etienne, Service de médecine nucléaire, Saint-Etienne, France

${ }^{3}$ CHRU Nancy, Service de médecine nucléaire, Nancy, France

${ }^{4}$ Centre Hospitalier Alpes Léman, Service de Médecine Nucléaire, Contamine sur Arve, France

${ }^{5}$ Centre Jean Perrin, Service de Médecine Nucléaire, Clermont Ferrand, France

${ }^{6}$ Groupe Hospitalier de 1’Est, Service de Médecine Nucléaire, Bron, France

${ }^{7}$ Hôpital Foch, Service de Médecine Nucléaire, Suresne, France

${ }^{8}$ CHU de Nantes, Service de médecine nucléaire, Nantes, France

${ }^{9}$ Centre d'Imagerie Fonctionnelle, Bordeaux, France

${ }^{10}$ Hôpital Lariboisière, APHP, Service de médecine nucléaire, Paris, France

${ }^{11}$ CHU Nice, Service de medecine Nucléaire, Nice, France

${ }^{12}$ Centre d'Imagerie Nucléaire, Annecy, France

No conflict of interest to be declared 
First/corresponding author

Dr Pierre-Yves Le Roux

Service de médecine nucléaire, CHRU de Brest, 29609 Brest Cedex, France

Phone number:+33298347892

Fax number:+33298347898

e-mail: pierre-yves.leroux@chu-brest.fr

Manuscript word counts: 4332

Running foot line: V/P scintigraphy in COVID-19 patient

Immediate Open Access: Creative Commons Attribution 4.0 International License (CC BY) allows users to share and adapt with attribution, excluding materials credited to previous publications.

License: https://creativecommons.org/licenses/by/4.0/.

Details: https://jnm.snmjournals.org/page/permissions.

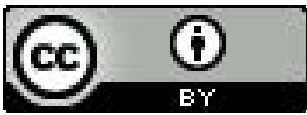




\begin{abstract}
In patients with novel coronavirus disease 2019 (COVID-19) referred for lung scintigraphy for suspected pulmonary embolism $(\mathrm{PE})$, there has been an ongoing debate within the nuclear medicine community as to whether and when the ventilation study should be performed. Indeed, while PE diagnosis typically relies on the recognition of ventilation/perfusion (V/P) mismatched defects, the ventilation procedure potentially increases the risk of contamination to the healthcare workers. The primary aim of this study was to assess the role of ventilation imaging when performing lung scintigraphy for suspected PE in COVID-19 patients. The secondary aim was to describe practices and imaging findings in this specific population.

Methods: A national registry was created in collaboration with the French Society of Nuclear Medicine to collect lung scans performed in COVID-19 patients for suspected PE. Practices of departments were assessed regarding imaging protocols and aerosol precautions. A retrospective review of V/P SPECT/CT scans was then conducted. Two physicians blinded to clinical information reviewed each case by sequentially using $\mathrm{P}$ SPECT, P SPECT/CT and V/P SPECT/CT images. Scans were classified in one of the four following categories: patients for whom PE could reasonably be excluded based on 1) perfusion SPECT only, 2) P SPECT/CT, 3) V/P SPECT/CT; or 4) patients with mismatched defects suggestive of PE according to the EANM criteria.
\end{abstract}

Results: Data from 12 French nuclear medicine departments were collected. Lung scans were performed between 03/2020 and 04/2021. Personal protective equipment and dedicated cleaning procedures were used in all departments. Out of the $145 \mathrm{~V} / \mathrm{Q}$ SPECT/CT included in the central review, PE could be excluded using only P SPECT, P SPECT/CT and V/P SPECT/CT in 27 (19\%), 55 (38\%) and 45 (31\%) patients, respectively. V/P SPECT/CT was positive for PE in 18 (12\%) patients, including $12(67 \%)$ with a low burden of PE $(\leq 10 \%)$.

Conclusion: In this population of COVID-19 patients assessed with lung scintigraphy, PE could be confidently excluded without ventilation in only $57 \%$ of patients. Ventilation imaging was required to confidently rule out PE in $31 \%$ of patients. Overall, the prevalence of PE was low (12\%).

Key words: pulmonary embolism; ventilation perfusion scintigraphy; single photon emission computed tomography; COVID-19 


\section{TEXT}

\section{INTRODUCTION}

A frequent complication of novel coronavirus disease 2019 (COVID-19) is coagulopathy, that manifests in the form of both micro-thrombosis and venous thromboembolism (1). Lung ventilation/perfusion (V/P) scintigraphy is a well-established test for pulmonary embolism (PE) diagnosis (2). Diagnostic strategies based on lung scintigraphy have been widely validated in large diagnostic accuracy (3) and management outcome studies (4-7), in which the lung scan was interpreted based on the recognition of wedge shaped perfusion defects, i.e. perfusion defects with normal ventilation.

However, the ventilation procedure increases the potential risk of contamination by the aerosol secretion and the expired air to healthcare workers and to other patients $(8,9)$. As a result, a variety of strategies have been proposed in the nuclear medicine literature regarding performance of lung scintigraphy in COVID-19 patients with suspected acute PE (10). Some have proposed to omit the ventilation scan and to only perform a perfusion scintigraphy or a perfusion SPECT/CT (11-14), arguing that this approach allows sufficient

diagnostic performances while reducing the risk of contamination. Others have recommended to systematically maintain standard V/P procedure, with appropriate aerosol precautions for healthcare workers (15-17). The rational for this approach is that a wide proportion of patients with confirmed COVID-19 infection and symptoms suggestive of acute PE will have an abnormal perfusion scan and that not performing a ventilation scan is associated to an unacceptably high risk of false positive results. Finally, some have proposed an intermediate approach with a standalone perfusion planar or SPECT/CT scan, followed, only when necessary, by a ventilation scan $(18,19)$. However, while various conflicting opinions and recommendations have been published on the performance of lung scintigraphy in COVID-19 patients, there is currently no factual data in this specific population to support recommendations to the nuclear medicine community.

The primary aim of this study was to assess the role of ventilation imaging when performing lung scintigraphy for suspected PE in COVID-19 patients. The secondary aim was to describe practices and imaging findings in this specific population. 


\section{MATERIALS AND METHODS}

\section{Collection Of Data}

A national registry was created at the initiative of the French Society of Nuclear Medicine working group on lung scintigraphy to collect lung scans performed in COVID-19 patients for suspected PE. In collaboration with the French Society of Nuclear Medicine, the information was circulated to its memberships via 3 sets of e-mails in April, June and December 2020. Physicians who have notified cases were then contacted to participate to the study. The protocol was approved by the nuclear medicine research ethics committee (CEMEN 2021-01). Informed consent was obtained from all participants.

\section{Evaluation Of Practices}

General practices of nuclear medicine facilities were assessed using a standardized questionnaire. The questionnaire encompassed aerosol precautions for the healthcare workers (type of mask, goggles, gloves, long cap, gown), cleaning procedures, organizational adaptation in the nuclear medicine facility, and imaging protocols for the performance of lung scintigraphy in patients with confirmed COVID-19 disease and suspected acute PE. Nuclear medicine facilities were also questioned on any possible contamination of a healthcare worker after managing a patient with COVID-19 disease.

Individual data from patients with confirmed COVID-19 disease who underwent lung scintigraphy for suspected acute PE were then collected. This includes patients who underwent various acquisition protocols, including planar scintigraphy (with or without ventilation), P SPECT/CT (without ventilation) or V/P SPECT/CT scans. Only patients still considered contagious were analyzed, i.e. patients for whom the lung scan was typically performed within 14 days after initiation of symptoms. For each patient, demographic information and lung scan images were collected.

\section{V/P SPECT/CT Central Review}

In order to assess the role of ventilation imaging in COVID-19 patients with suspected PE, a centralized retrospective review of scans with a complete V/P SPECT/CT protocol was then conducted. Scans of patients who underwent a planar or a P SPECT/CT protocol (without ventilation) were not included in this 
retrospective review. A consensus reading of images was performed by two board-certified nuclear medicine physicians blinded to clinical information. Each case was reviewed by sequentially using P SPECT, P SPECT/CT and V/P SPECT/CT images, respectively. Firstly, only P SPECT images were used. Scans with normal perfusion were classified as negative for PE. Secondly, in patients with an abnormal P SPECT, CT images were added. Scans whose perfusion defects were matched with CT findings were classified as negative for PE. Finally, in patients with mismatched P SPECT/CT defects, V SPECT images were added for interpretation. V/P SPECT/CT scans were then interpreted as negative or positive for PE according to the presence of V/P mismatched defects suggestive of acute PE according to the EANM criteria $(20,21)$. Accordingly, scans were classified in one of the four following categories: patients for whom PE could reasonably be excluded based on 1) perfusion SPECT only, 2) P SPECT/CT, 3) V/P SPECT/CT; or 4) patients with mismatched V/P defects suggestive of PE. In patients with a positive V/Q SPECT/CT, the number of segmental and sub-segmental mismatched defects was recorded.

\section{RESULTS}

Data from 183 patients with confirmed COVID-19 disease who underwent lung scintigraphy for suspected acute PE between 03/2020 and 04/2021 in 12 French nuclear medicine departments were collected.

\section{Evaluation Of Practices}

Practices of the 12 nuclear medicine departments are presented in Table 1. Personal protective equipment and dedicated cleaning and disinfecting procedures were used in all departments. Scans were formed in a dedicated room in $8(67 \%)$ centers, and at the end of the day in $10(83 \%) .11(92 \%)$ of centers systematically performed a ventilation scan before the perfusion acquisition. V/P SPECT/CT was the first line imaging procedure in $8(75 \%)$ centers. Out of the 12 nuclear medicine facilities, there was no reported case of possible contamination of a healthcare worker after managing a COVID-19 patient.

Out of the 183 patients, 117 (64\%) were female. Median age was 74 (15-102) years old. 68 (37\%) were older than 80 , and $26(14 \%)$ were older than 90.168 patients $(92 \%)$ had a ventilation scan, using ${ }^{99 \mathrm{~m}} \mathrm{Tc}-\mathrm{Technegas}$ 
aerosol in $144(86 \%)$ patients and ${ }^{81}$ Krypton gas in $24(14 \%)$ patients, respectively. 24 (13\%) only had a planar scan and $159(87 \%)$ had SPECT/CT imaging.

\section{V/Q SPECT/CT Interpretation}

145 complete V/P SPECT/CT scans were centrally reviewed. Out of them, PE could be excluded using only P SPECT, P SPECT/CT and V/P SPECT/CT in 27 (19\%), 55 (38\%) and 45 (31\%) patients, respectively (See Figure 1). Examples of negative P SPECT/CT scans with perfusion defects matched with chest CT findings of COVID-19 disease are shown in Figure 2. Examples of false positive results using the P SPECT/CT approach are presented in Figure 3. V/P SPECT/CT imaging was positive for PE in 18 (12\%) patients. The burden of PE was $\leq 10 \%$ in $12(67 \%)$ patients, $>10 \%$ but $\leq 20 \%$ in $4(22 \%)$ patients, and $>20 \%$ in $2(11 \%)$ patients, respectively. Examples of positive V/P SPECT/CT scans are shown in Figure 4.

\section{DISCUSSION}

In this population of COVID-19 patients assessed with lung scintigraphy, PE could be confidently excluded without ventilation in $57 \%$ of patients. In contrast, ventilation imaging was required in the remaining $43 \%$ of patients, to confidently rule out $(31 \%)$ or confirm (12\%) PE diagnosis.

Lung scan interpretation for PE diagnosis typically relies on the recognition of ventilation/perfusion (V/P) mismatched defects (22). The sensitivity of the test, i.e. the ability to detect PE, relies on the recognition of defect on perfusion images. A normal perfusion scan has been shown to safely rule out PE diagnosis (4-7). However, there are many 'nonthromboembolic' lung diseases that may cause perfusion defects. In order to increase the specificity, i.e. the ability of the test to detect that a patient is free of disease, perfusion scintigraphy has to be compared to ventilation images to differentiate V/P mismatched defects highly suggestive of acute PE from V/P matched defect from other etiology. With the advent of SPECT/CT cameras, it has been proposed to co-register SPECT data to a low dose CT to further increase the specificity of the test, or by some groups to replace the ventilation SPECT. 
In our series, only 19\% of COVID-19 patients had a strictly normal perfusion SPECT scan. In this specific population of COVID-19 patients with suspected acute PE, there is therefore a high likelihood (nearly 4 out of 5 times) that an approach consisting in only performing a perfusion lung scan, without CT images, will be non-diagnostic. When co-registering perfusion SPECT images with a low dose CT scan, 38\% more scans became diagnostic as all perfusion defects could be confidently explained by non PE morphological findings. Overall, P SPECT/CT imaging would have allowed to confidently exclude PE in 57\% of COVID-19 patients, without exposing the healthcare workers to an increased risk of contamination due to the ventilation procedure. Nevertheless, the benefit of omitting the ventilation should be balanced against the risk of increasing transfers of patients through the healthcare facilities and the number of contact between individuals.

In the remaining $43 \%$ patients, a ventilation scan was required to exclude $(31 \%)$ or confirm $(12 \%)$ the diagnosis of PE. Of note is that most of patients with a positive scan did not have massive PE with multiple wedge shaped perfusion defects highly suggestive of PE irrespective of the ventilation scan. On the contrary, the ventilation study was helpful in most of positive scans that only demonstrated 1 or 2 segmental perfusion mismatched defects.

Our data confirms the high risk of a false positive result when omitting the ventilation scan. In a retrospective series of 393 patients assessed by V/P SPECT imaging for suspected PE, 15\% of patients with a negative V/P SPECT would have been wrongly diagnosed with PE using a P SPECT/CT approach (23). In another series of 81 patients, specificity of decreased from $100 \%$ with V/P SPECT/CT to $51 \%$ with a P SPECT/CT (24). Similarly, in a study of 93 patients, $17 \%$ of negative V/Q SPECT were falsely positive with P SPECT/CT imaging (25). The risk of false positive results was even higher in our population of COVID-19 patients. This is likely due to the fact that a large majority of patients had COVID-19 parenchymal lung disease and thus abnormal lung perfusion. Also noteworthy is the age of the population, with a median age of 74 years old and $37 \%$ of patients older than 80 , which increases the likelihood of abnormal perfusion from any lung disease.

A false positive diagnostic test may have major consequences for patients with suspected acute PE. Indeed, current clinical guidelines suggest extended anticoagulation in patients with no identifiable risk factor or with 
a minor transient/reversible risk factor for the index PE event (2). Accordingly, a false positive lung scintigraphy will mean lifelong anticoagulant therapy and its risks of bleeding for many patients (15).

Surprisingly, the prevalence of positive scans was low (12\%) in this population of COVID-19 patients with suspected PE assessed with V/Q SPECT/CT imaging. This is much lower than the prevalence of PE across studies on V/Q SPECT in non-COVID-19 patients, that ranges from $17 \%$ to $54 \%$ (median $26 \%$ ) (26). This is also low as compared with studies assessing other imaging test for PE (e.g. CT pulmonary angiography or planar V/P scan). In a meta-analysis performed before the COVID-19 pandemic including 29684 patients from 49 studies, the pooled prevalence of PE was $22.6 \%$ (27). As it is now well established that COVID-19 predisposes patients to thrombotic events (28), this likely means that there are more PE suspicions in COVID19 patients than in non COVID-19 patients, likely resulting from the combination of poorly explained respiratory symptoms in patients at risk for venous thromboembolism. Furthermore, in patients positive for PE, the burden of disease was low, with only $11 \%$ of patients with a PE burden $>20 \%$. It is likely that most of patients admitted to intensive care unit or with signs of haemodynamic instability were referred to CT pulmonary angiography rather than to lung scintigraphy (29).

Assessment of general practices showed a major trend among the 12 French institutions. The typical examination was a V/P SPECT/CT acquisition, as commonly performed in non Covid-19 patients (30), with personal protective equipment for the healthcare workers and dedicated cleaning procedures. Most of institutions performed acquisitions on a dedicated camera at the end of the day. Although it should be interpreted with caution, no case of possible contamination of a healthcare worker after managing a COVID19 patient was reported.

Our study has some limitations. First, the results of the central review were not compared to an independent reference standard and patient's follow-up was not collected. Accordingly, we cannot assert that all patients with a negative scan did not have PE and that all patients with a positive scan did have PE. Despite these limitations resulting from the retrospective design of this multicentre study based on a national registry, this is 
the first large series that assessed the usefulness of the ventilation scan in COVID-19 patients. Major trends have emerged with regards to the prevalence of $\mathrm{PE}$ and the proportion of inconclusive results with the various lung scan protocols. These results may assist nuclear physicians in the performance of lung scintigraphy in COVID-19 patients with suspected acute PE, according to their own local situation. Second, assessment of general practices probably does not reflect the reality in nuclear medicine facilities around the world. Indeed, the French Society of Nuclear Medicine working group on lung scintigraphy recommended the performance of a ventilation scan in COVID-19 patients, which is likely to have influenced practices in France. Furthermore, institutions that followed these recommendations may have been more inclined to participate to the study. However, our results show that the usual V/P SPECT/CT approach can be used in daily practice in COVID-19 patients. In our series, there was no reported case of possible contamination of a healthcare worker after managing a COVID-19 patient. However, this was just observational and we did not perform formal testing such as swabbing for virus. Accordingly, we cannot draw any conclusions on the risk of contamination. Third, according to the EANM guidelines for lung scintigraphy (20) or the French Society of Nuclear Medicine guideline for lung scintigraphy protocols (31), the CT were performed as a low-dose CT scan during continuous shallow breathing. Accordingly, the CT scans do not fulfill quality criteria to be diagnostic. Optimization of acquisition and reconstruction parameters could enhance the diagnostic performance of the CT scans. Finally, we performed a consensual interpretation of scans and did not assess the interobserver agreement.

\section{CONCLUSION}

In COVID-19 patients assessed with lung scintigraphy, PE could be confidently excluded without ventilation in $57 \%$ of patients. This approach allows to limit the risk of contamination to the healthcare workers, but should be balanced against the risk of increasing transfers of patients through the healthcare facilities in the remaining $43 \%$ patients. Indeed, our study clearly confirms the high risk of false positive results when omitting the ventilation study, which appears inacceptable given the risk of bleeding and the trend towards indefinite duration of anticoagulation in many patients. 
Strategies should be adapted to each local situation but providing the best imaging test available should remain the priority. Ruling out PE diagnosis without the use of a ventilation scan is likely safe. However, confirming PE diagnosis requires the performance of a ventilation scan. Otherwise, the referral physician should be advised of the risk of false positive result.

\section{FINANCIAL DISCLOSURE}

No conflict of interest to be declared

\section{AKNOWLEDGEMENT}

The authors would like to acknowledge the French Society of Nuclear Medicine for its collaboration. The authors also acknowledge Doxaca, and especially Sylver Imhoff, for their assistance for managing the central review.

\section{KEY POINTS}

Question: In COVID-19 patients assessed with lung scintigraphy for suspected acute pulmonary embolism $(\mathrm{PE})$, what is the role of the ventilation scan?

Pertinent finding: In a retrospective review of 145 V/P SPECT/CT scans of COVID-19 patients referred for suspected acute PE, PE could be confidently excluded without ventilation in only $57 \%$ of patients. In contrast, ventilation imaging was required to confidently rule out PE in $31 \%$ of patients, confirming the high risk of false positive results when omitting the ventilation study

Implication for patent care:

In COVID-19 patients, confirming PE diagnosis with lung scintigraphy requires the performance of a ventilation scan. 


\section{REFERENCES}

1. Moores LK, Tritschler T, Brosnahan S, et al. Prevention, Diagnosis, and Treatment of VTE in Patients With Coronavirus Disease 2019: CHEST Guideline and Expert Panel Report. Chest. 2020;158(3):1143-1163.

2. Konstantinides SV, Meyer G, Becattini C, et al. 2019 ESC Guidelines for the diagnosis and management of acute pulmonary embolism developed in collaboration with the European Respiratory Society (ERS): The Task Force for the diagnosis and management of acute pulmonary embolism of the European Society of Cardiology (ESC). Eur Respir J. 2019;54(3).

3. Investigators P. Value of the ventilation/perfusion scan in acute pulmonary embolism. Results of the prospective investigation of pulmonary embolism diagnosis (PIOPED). JAMA. 1990;263(20):2753-2759.

4. Perrier A, Desmarais S, Miron MJ, et al. Non-invasive diagnosis of venous thromboembolism in outpatients. Lancet. 1999;353(9148):190-195.

5. Wells PS, Ginsberg JS, Anderson DR, et al. Use of a clinical model for safe management of patients with suspected pulmonary embolism. Ann Intern Med. 1998;129(12):997-1005.

6. Anderson DR, Kahn SR, Rodger MA, et al. Computed tomographic pulmonary angiography vs ventilation-perfusion lung scanning in patients with suspected pulmonary embolism: a randomized controlled trial. JAMA. 2007;298(23):2743-2753.

7. Salaun PY, Couturaud F, Le Duc-Pennec A, et al. Noninvasive diagnosis of pulmonary embolism. Chest. 2011;139(6):1294-1298.

8. Williams DA, Carlson C, McEnerney K, et al. Technetium-99m DTPA aerosol contamination in lung ventilation studies. J Nucl Med Technol. 1998;26(1):43-44.

9. Brudecki K, Borkowska E, Gorzkiewicz K, et al. (99m)Tc activity concentrations in room air and resulting internal contamination of medical personnel during ventilation-perfusion lung scans. Radiat Environ Biophys. 2019;58(3):469-475.

10. Zuckier LS. To everything there is a season: taxonomy of approaches to the performance of lung scintigraphy in the era of COVID-19. Eur J Nucl Med Mol Imaging. 2021;48(3):666-669.

11. Lu Y, Macapinlac HA. Perfusion SPECT/CT to diagnose pulmonary embolism during COVID-19 pandemic. Eur J Nucl Med Mol Imaging. 2020;47(9):2064-2065.

12. Tulchinsky M, Osmany S. The American College of Nuclear Medicine Guidance on Operating Procedures for a Nuclear Medicine Facility During COVID-19 Pandemic. Clin Nucl Med. 2021;46(7):571574.

13. Burger IA, Niemann T, Patriki D, et al. Is there a role for lung perfusion [(99m)Tc]-MAA SPECT/CT to rule out pulmonary embolism in COVID-19 patients with contraindications for iodine contrast? Eur J Nucl Med Mol Imaging. 2020;47(9):2062-2063. 
14. Das JP, Yeh R, Schoder H. Clinical utility of perfusion (Q)-single-photon emission computed tomography (SPECT)/CT for diagnosing pulmonary embolus (PE) in COVID-19 patients with a moderate to high pre-test probability of PE. Eur J Nucl Med Mol Imaging. 2021;48(3):794-799.

15. Le Roux PY, Le Gal G, Salaun PY. Lung scintigraphy for pulmonary embolism diagnosis during the COVID-19 pandemic: does the benefit-risk ratio really justify omitting the ventilation study? Eur J Nucl Med Mol Imaging. 2020;47(11):2499-2500.

16. BELNUC. Recommendations for performing V/Q scans in the context of COVID19. 2020.

17. perfusionnelles. GDTSEPve. Recommandations pour la réalisation de la scintigraphie pulmonaire dans le contexte d'épidémie due au virus Covid-19. 2020.

18. Zuckier LS, Moadel RM, Haramati LB, et al. Diagnostic Evaluation of Pulmonary Embolism During the COVID-19 Pandemic. J Nucl Med. 2020;61(5):630-631.

19. Schaefer WM, Knollmann D, Meyer PT. V/Q SPECT/CT in the Time of COVID-19: Changing the Order to Improve Safety Without Sacrificing Accuracy. J Nucl Med. 2021;62(7):1022-1024.

20. Bajc M, Schumichen C, Gruning T, et al. EANM guideline for ventilation/perfusion single-photon emission computed tomography (SPECT) for diagnosis of pulmonary embolism and beyond. Eur J Nucl Med Mol Imaging. 2019;46(12):2429-2451.

21. Le Roux PY, Robin P, Delluc A, et al. V/Q SPECT interpretation for pulmonary embolism diagnosis: which criteria to use? J Nucl Med. 2013;54(7):1077-1081.

22. Le Roux PY, Robin P, Salaun PY. New developments and future challenges of nuclear medicine and molecular imaging for pulmonary embolism. Thromb Res. 2018;163:236-241.

23. Le Roux PY, Robin P, Delluc A, et al. Additional value of combining low-dose computed tomography to V/Q SPECT on a hybrid SPECT-CT camera for pulmonary embolism diagnosis. Nucl Med Commun. 2015;36(9):922-930.

24. Gutte H, Mortensen J, Jensen CV, et al. Detection of pulmonary embolism with combined ventilation-perfusion SPECT and low-dose CT: head-to-head comparison with multidetector CT angiography. J Nucl Med. 2009;50(12):1987-1992.

25. Palmowski K, Oltmanns U, Kreuter M, et al. Diagnosis of pulmonary embolism: conventional ventilation/perfusion SPECT is superior to the combination of perfusion SPECT and nonenhanced CT. Respiration. 2014;88(4):291-297.

26. Le Roux PY, Robin P, Tromeur C, et al. Ventilation/perfusion SPECT for the diagnosis of pulmonary embolism: A systematic review. J Thromb Haemost. 2020;18(11):2910-2920.

27. Dronkers CEA, van der Hulle T, Le Gal G, et al. Towards a tailored diagnostic standard for future diagnostic studies in pulmonary embolism: communication from the SSC of the ISTH. $J$ Thromb Haemost. 2017;15(5):1040-1043.

28. Schulman S, Hu Y, Konstantinides S. Venous Thromboembolism in COVID-19. Thromb Haemost. 2020;120(12):1642-1653. 
29. Bompard F, Monnier H, Saab I, et al. Pulmonary embolism in patients with COVID-19 pneumonia. Eur Respir J. 2020;56(1).

30. Le Roux PY, Pelletier-Galarneau M, De Laroche R, et al. Pulmonary Scintigraphy for the Diagnosis of Acute Pulmonary Embolism: A Survey of Current Practices in Australia, Canada, and France. J Nucl Med. 2015;56(8):1212-1217.

31. Le Roux PY, Blanc-Béguin F, Bonnefoy PB, et al. Guide pour la rédaction de protocoles pour la scintigraphie pulmonaire. Médecine Nucléaire. 2021;45(2):85-92. 


\section{FIGURES}

Figure 1

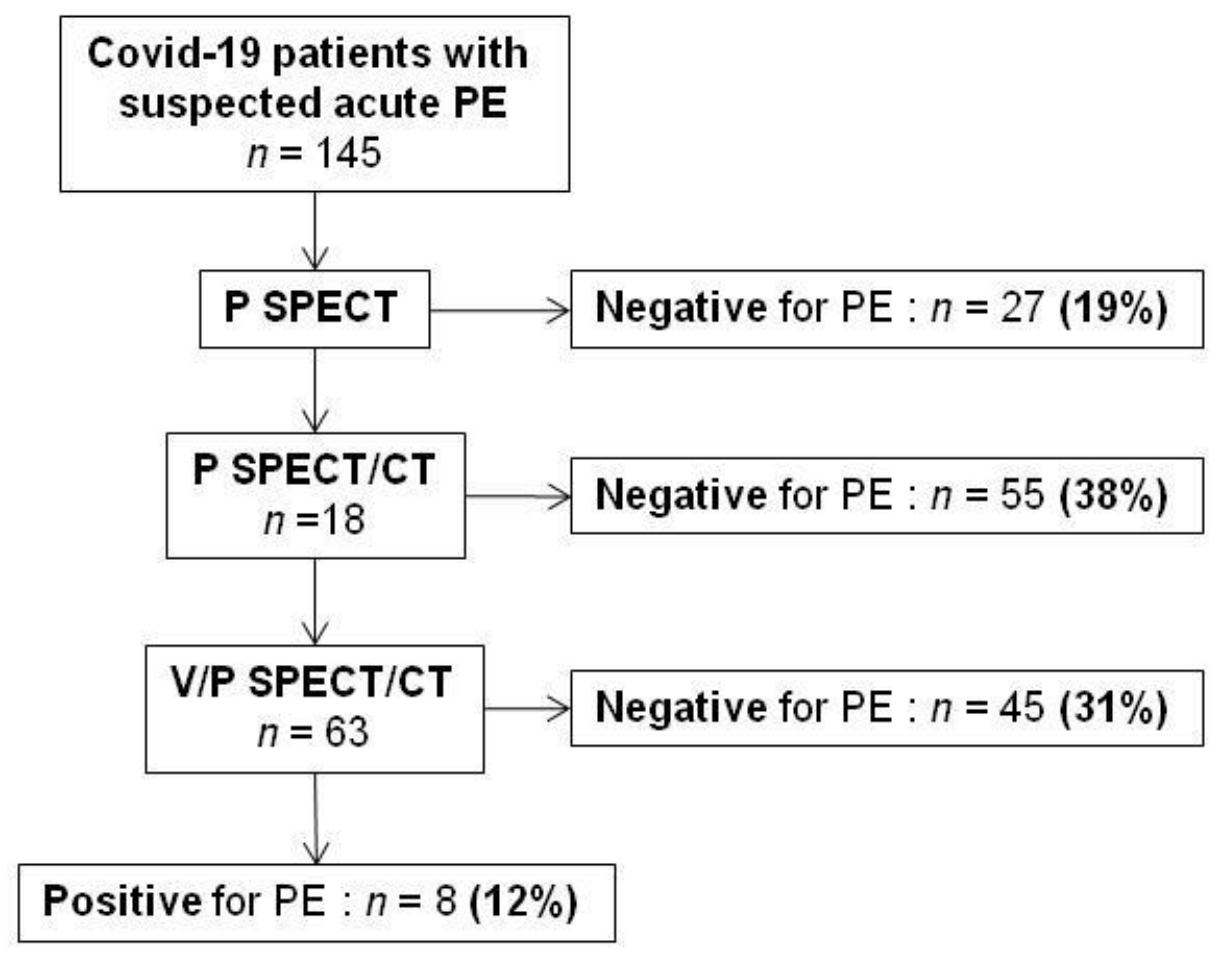

Figure 1: Results of the central review 
Figure 2
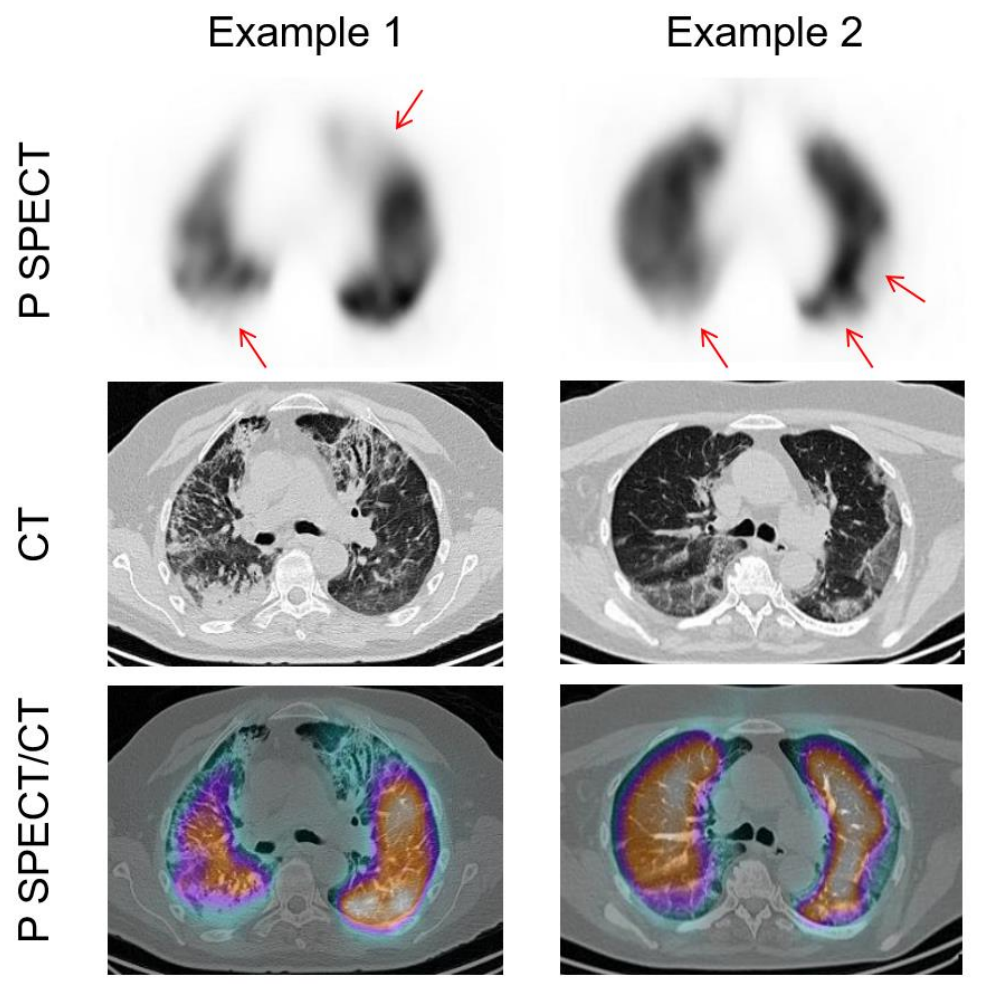

\section{Example 3}
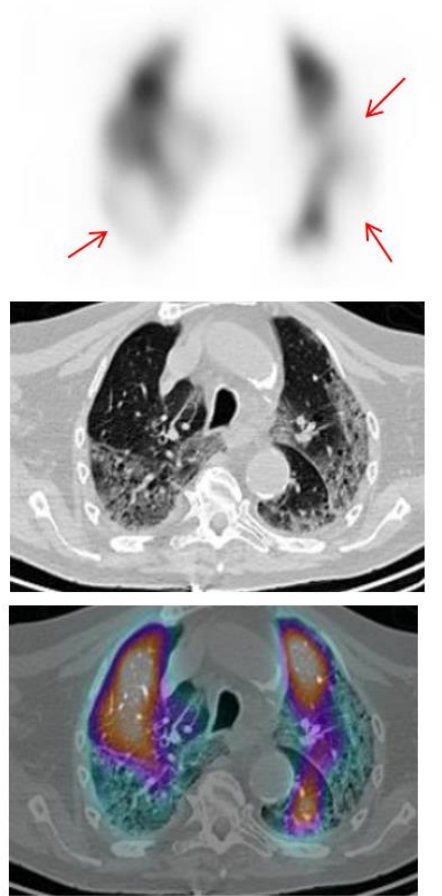

Figure 2: Examples of negative P SPECT/CT scans, with perfusion defects matched with chest CT findings of COVID-19 disease. 
Figure 3

\section{Example 1}

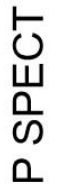
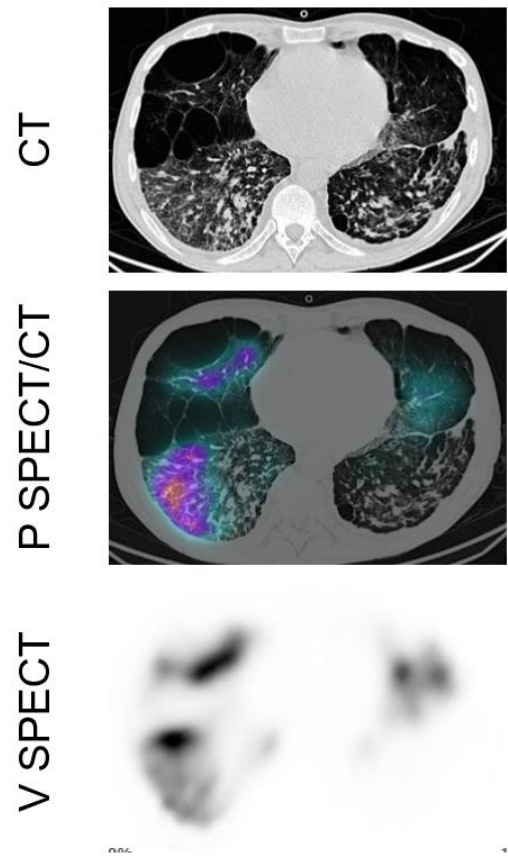

Example 2
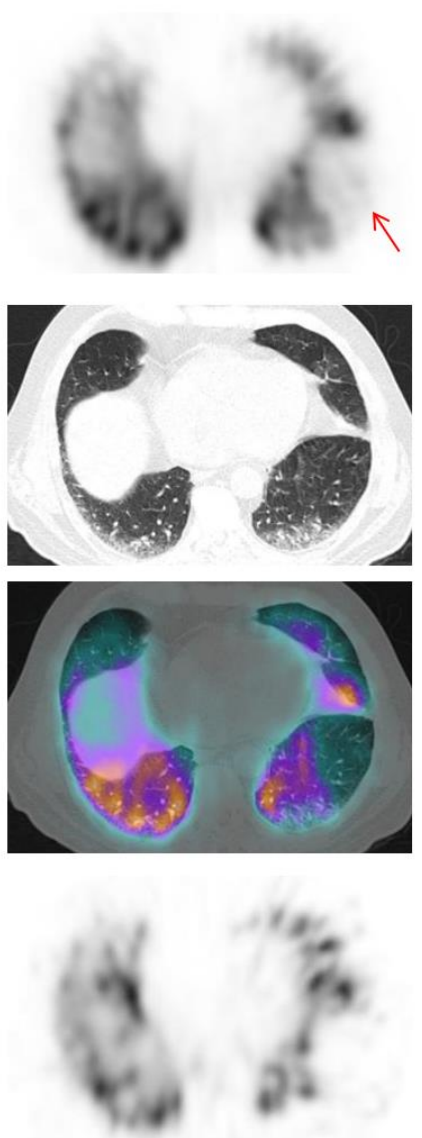

Example 3
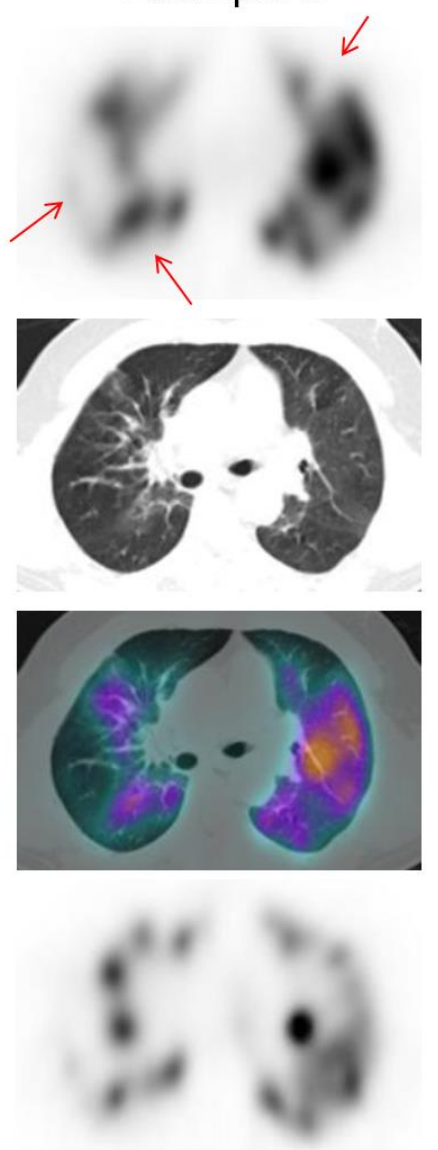

Figure 3: Examples of false positive result using the P SPECT/CT approach.

Perfusion SPECT images showed perfusion defects, without significant abnormality on CT images. P

SPECT/CT scans would therefore have been read as positive for PE. However, V SPECT demonstrated matched defects. V/Q SPECT/CT scans were therefore interpreted as negative for PE. 
Figure 4

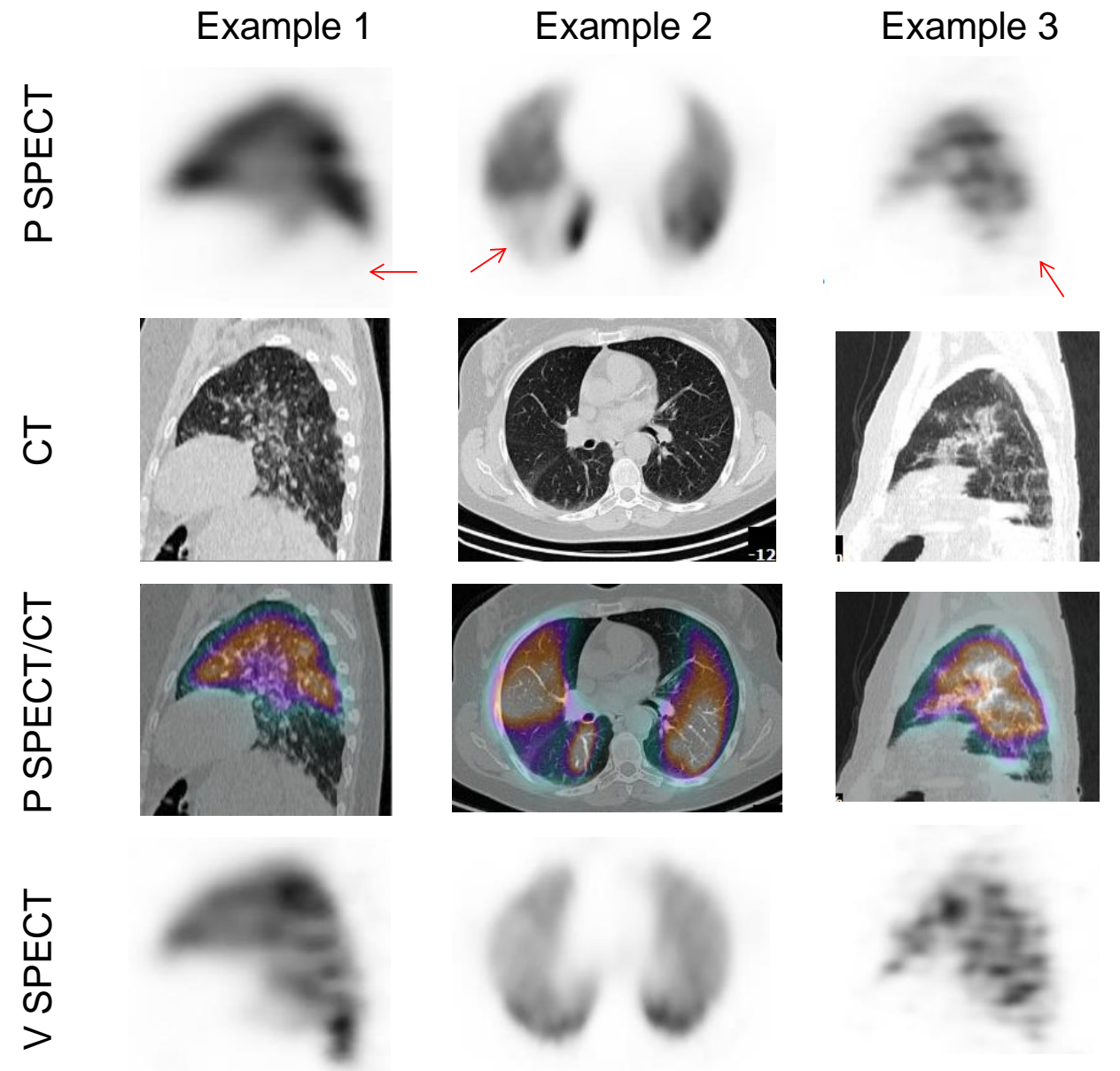

Figure 4: Examples of positive V/P SPECT/CT scans.

P SPECT images showed perfusion defects while co-registered V SPECT images demonstrated normal ventilation (mismatched V/P defects). 
Table 1: Practices of nuclear medicine facilities

\begin{tabular}{|c|c|}
\hline Practices & No of centres $(\%)$ \\
\hline Aerosol precautions & $12(100)$ \\
\hline Mask & $12(100)$ \\
\hline FFP1 & $1(8)$ \\
\hline FFP2 & $11(92)$ \\
\hline Goggles & $12(100)$ \\
\hline Long cap & $12(100)$ \\
\hline Gloves & $12(100)$ \\
\hline Gown & $12(100)$ \\
\hline Specific COVID-19 cleaning and disinfecting procedures & $12(100)$ \\
\hline \multicolumn{2}{|l|}{ Organizational adaptation in the nuclear medicine facility } \\
\hline Camera/room dedicated to COVID-19 patients & $8(67)$ \\
\hline Scans performed at the end of the day & $10(83)$ \\
\hline Dedicated circuit within the nuclear medicine department & $3(25)$ \\
\hline \multicolumn{2}{|l|}{ Standard lung scan protocol for COVID-19 patients } \\
\hline Systematic ventilation scan & $11(92)$ \\
\hline Technegas-Tc ${ }^{99 m}$ & $8(73)$ \\
\hline Krypton $^{81 \mathrm{~m}}$ gas & $3(27)$ \\
\hline \multicolumn{2}{|l|}{ First line imaging Planar / SPECT } \\
\hline Planar & $3(25)$ \\
\hline SPECT & $0(0)$ \\
\hline SPECT/CT & $9(75)$ \\
\hline FFP : filtering facepiece & \\
\hline
\end{tabular}


Graphical Abstract

\section{Lung scintigraphy for pulmonary embolism diagnosis in COVID-19 patients}

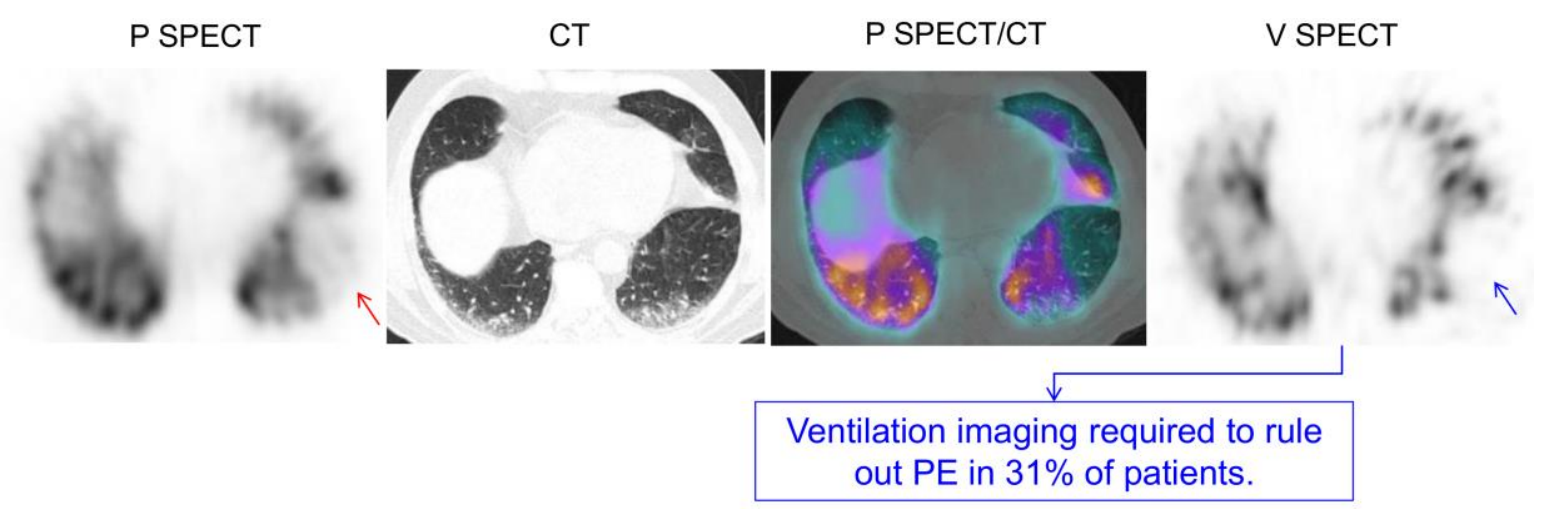

Implications: In COVID-19 patients, confirming PE diagnosis with lung scintigraphy requires the performance of a ventilation scan. 